\title{
Shifting energy metabolism
}

A cell's ability to adjust its rate of glycolysis relative to oxidative metabolism allows it to adapt to changes in nutrient availability and energy demands. Targeting such shifts could be therapeutically useful in diseases like cancer, in which tumour cells rely more heavily on glycolysis, or in ischaemia and stroke, in which there is an increase in mitochondrial respiration as oxygen availability becomes limited. Mootha and colleagues present a nutrientsensitized screening assay to identify agents that can shift cell metabolism, and demonstrate its application to uncover new roles for approved drugs that had not previously been linked to energy metabolism.

The authors compared the effects of more than 3,500 small molecules on the growth and proliferation of fibroblasts cultured in either glucoseor galactose-containing media. As expected, known chemotherapeutic agents inhibited the rapid proliferation of cells in glucose, whereas agents that are known to inhibit the mitochondrial respiratory chain - for example, the antimalarials mefloquine and artemisinin — inhibited the growth of cells in galactose-containing media (which rely more heavily on mitochondrial respiration for ATP production).
Interestingly, meclizine, a non-prescription drug for nausea and vertigo, redirected oxidative metabolism towards glycolysis. This effect did not seem to depend on its established antagonistic effects on histamine or muscarinic acetylcholine receptors, and was not observed in isolated mitochondria. These findings suggest that meclizine inhibits mitochondrial respiration indirectly, although a transcriptional mechanism is unlikely, as no changes in the stability of hypoxia-inducible factors were observed.

As agents that inhibit mitochondrial respiration have been shown to confer protection against stroke and myocardial infarction, the authors tested whether meclizine could protect rat cardiomyocytes in an ex vivo model of ischaemic injury and in primary mouse neurons following transient middle artery cerebral occlusion. In both cases, prior administration of well-tolerated meclizine doses reduced the area of infarction, suggesting a potential novel indication for this drug.

Although intervening in energy metabolism is therapeutically promising, there are a limited number of agents that have the potential to safely achieve this goal at present. This study shows how a

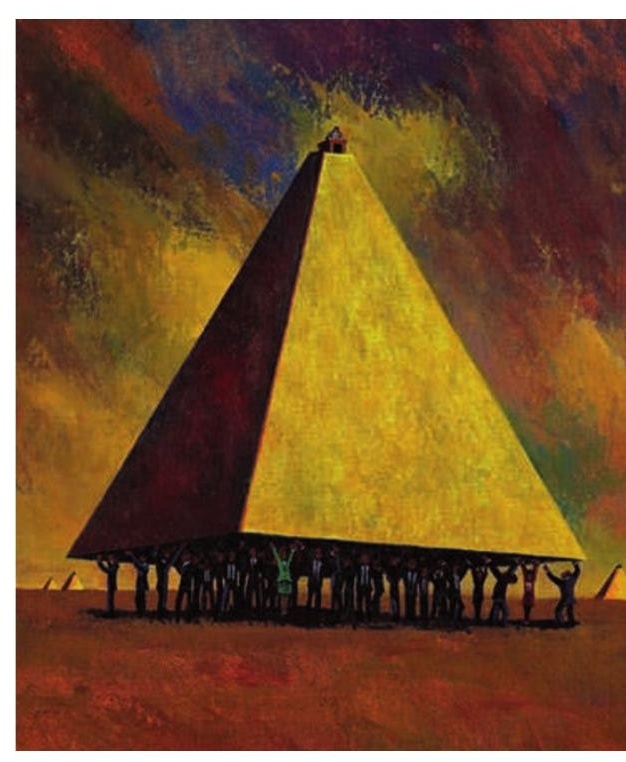

simple screening assay could be used to expand the arsenal of such agents, on the one hand, identifying lead compounds for prophylactic treatments for ischaemia-reperfusion, and on the other, agents that could be of value in cancer and other proliferative diseases.

\section{Monica Hoyos Flight}

ORIGINAL RESEARCH PAPER Gohil, V. M. et al. Nutrient-sensitized screening for drugs that shift energy metabolism from mitochondrial respiration to glycolysis. Nature Biotech. 28 , 249-255 (2010) 